\title{
TrES-1: The Transiting Planet of a Bright KO V Star
}

\section{Citation}

Alonso, Roi, Timothy M. Brown, Guillermo Torres, David W. Latham, Alessandro Sozzetti, Georgi Mandushev, Juan A. Belmonte, et al. 2004. "TrES-1: The Transiting Planet of a Bright KO V Star." The Astrophysical Journal 613 (2): L153-56. https://doi.org/10.1086/425256.

\section{Permanent link}

http://nrs.harvard.edu/urn-3:HUL.InstRepos:41397424

\section{Terms of Use}

This article was downloaded from Harvard University's DASH repository, and is made available under the terms and conditions applicable to Other Posted Material, as set forth at http:// nrs.harvard.edu/urn-3:HUL.InstRepos:dash.current.terms-of-use\#LAA

\section{Share Your Story}

The Harvard community has made this article openly available.

Please share how this access benefits you. Submit a story.

\section{Accessibility}


The Astrophysical Journal, 613:L153-L156, 2004 October 1

(C) 2004. The American Astronomical Society. All rights reserved. Printed in U.S.A.

TrES-1: THE TRANSITING PLANET OF A BRIGHT K0 V STAR

\author{
Roi Alonso, ${ }^{1,2}$ Timothy M. Brown, ${ }^{2}$ Guillermo Torres, ${ }^{3}$ David W. Latham, ${ }^{3}$ Alessandro Sozzetti, ${ }^{3,4}$ \\ Georgi Mandushev, ${ }^{5}$ Juan A. Belmonte, ${ }^{1}$ David Charbonneau, ${ }^{3,6}$ Hans J. Deeg, ${ }^{1}$ \\ Edward W. Dunham, ${ }^{5}$ Francis T. O’Donovan, ${ }^{6}$ and Robert P. Stefanik ${ }^{3}$ \\ Received 2004 August 6; accepted 2004 August 23; published 2004 September 1
}

\begin{abstract}
We report the detection of a transiting Jupiter-sized planet orbiting a relatively bright $(V=11.79) \mathrm{K} 0 \mathrm{~V}$ star. We detected the transit light-curve signature in the course of the TrES multisite transiting planet survey and confirmed the planetary nature of the companion via multicolor photometry and precise radial velocity measurements. We designate the planet TrES-1; its inferred mass is $(0.75 \pm 0.07) M_{\text {Jup }}$, its radius is $1.08_{-0.04}^{+0.18} R_{\text {Jup }}$, and its orbital period is $3.030065 \pm 0.000008$ days. This planet has an orbital period similar to that of HD 209458 b but about twice as long as those of the OGLE transiting planets. Its mass is indistinguishable from that of HD 209458b, but its radius is significantly smaller and fits the theoretical models without the need for an additional source of heat deep in the atmosphere, as has been invoked by some investigators for HD $209458 \mathrm{~b}$.

Subject headings: binaries: eclipsing — planetary systems — stars: individual (GSC 02652-01324) — techniques: photometric — techniques: radial velocities
\end{abstract}

\section{INTRODUCTION}

Since before the discovery of the first transiting extrasolar planet (Charbonneau et al. 2000; Henry et al. 2000), it has been recognized that transits provide a sensitive way to infer the existence of small bodies orbiting other stars (Struve 1952). There are now dozens of ground-based photometric searches underway that aim to detect planets of distant stars by means of their photometric signatures (Horne 2003) as well as several space projects with the same purpose (Auvergne et al. 2003; Borucki et al. 2003).

Until now, the only confirmed planet detections by transits (Konacki et al. 2003, 2004; Bouchy et al. 2004) have been based on the Optical Gravitational Lensing Experiment (OGLE) survey (Udalski et al. 2002a, 2002b, 2003), which is performed with a telescope of $1.3 \mathrm{~m}$ aperture. The strategy of using a moderateaperture telescope with seeing-limited spatial resolution must be commended for its obvious successes and moreover because the three OGLE planets are peculiar, having the shortest orbital periods yet known. But surveys using large telescopes suffer from the faintness of the stars with which they deal (the I magnitudes of the OGLE planet host stars range from 14.4 to 15.7). For such faint stars, the necessary follow-up observations are difficult and time-consuming, and the precision with which planetary parameters such as mass and radius can be determined is compromised.

For these reasons, we have pursued a transiting planet search organized along different lines-one that uses small-aperture, wide-field telescopes to search for transits among brighter stars. The principal challenge facing wide-field surveys such as ours is to attain adequate photometric precision in the face of spatially varying atmospheric extinction and instrumental effects. And,

\footnotetext{
${ }^{1}$ Instituto de Astrofísica de Canarias, 38200 La Laguna, Tenerife, Spain; ras@iac.es,jba@iac.es, hdeeg@iac.es.

${ }^{2}$ High Altitude Observatory/National Center for Atmospheric Research, 3450 Mitchell Lane, Boulder, CO 80307; timbrown@hao.ucar.edu.

${ }^{3}$ Harvard-Smithsonian Center for Astrophysics, 60 Garden Street, Cambridge, MA 02138; gtorres@cfa.harvard.edu, dlatham@cfa.harvard.edu, asozzett@ cfa.harvard.edu,dcharbonneau@cfa.harvard.edu,rstefanik@cfa.harvard.edu.

${ }^{4}$ Department of Physics and Astronomy, University of Pittsburgh, 100 Allen Hall, Pittsburgh, PA 15260.

${ }^{5}$ Lowell Observatory, 1400 West Mars Hill Road, Flagstaff, AZ 86001; gmand@lowell.edu,dunham@lowell.edu.

${ }^{6}$ California Institute of Technology, 1200 East California Boulevard, Pasadena, CA 91125; ftod@astro.caltech.edu.
}

as in all planet-search surveys, we must implement efficient methods for rejecting the many false alarms that appear in the photometric light curves. These false alarms result almost entirely from eclipsing systems involving two or more stars, including grazing eclipsing binaries, small stars transiting large ones, and eclipsing binaries diluted by the light of a third star. For bright-star searches, these imposters can outnumber true planetary transits by an order of magnitude (Brown 2003). Because of the diverse nature of the false alarm sources, several kinds of follow-up observations are needed to reject them all (Alonso et al. 2004). We report here the first transiting extrasolar planet to be detected by such a wide-field, bright-star survey. We also describe the confirmation process in some detail, as an illustration of the necessary steps in verifying that transits are caused by an object of planetary and not stellar mass.

\section{OBSERVATIONS}

Our initial photometric observations leading to the detection of a planetary transit signature were conducted using the three telescopes of the Trans-Atlantic Exoplanet Survey (TrES) network. These telescopes (STellar Astrophysics and Research on Exoplanets [STARE], located on Tenerife in the Canary Islands; Planet Search Survey Telescope [PSST], located at Lowell Observatory, Arizona; and Sleuth, located at Mount Palomar, California) ${ }^{7}$ are being described individually elsewhere (Dunham et al. 2004; T. M. Brown et al. 2004, in preparation). Briefly, all three are small-aperture $(10 \mathrm{~cm})$, wide-field $\left(6^{\circ}\right), \mathrm{CCD}$-based systems with spatial resolution of about $11^{\prime \prime}$ pixel $^{-1}$. They usually observe in red light (roughly Johnson $R$ for STARE and PSST, Sloan $r$ for Sleuth), and they operate in coordination, observing the same field in the sky continuously (or as nearly as possible) for typically 2 month intervals. The observing cadence at each site is roughly one image every 2 minutes, and the resulting time series are later binned to 9 minute time resolution. Recent adoption of an imagesubtraction algorithm (based on Alard 2000) yields photometric precision of better than 2 mmag for the brightest nonsaturating stars $(R \simeq 8)$ and better than $10 \mathrm{mmag}$ for $R \leq 12.5$.

We designate the planet described herein as TrES-1, the first

${ }^{7}$ See also http://www.hao.ucar.edu/public/research/stare/stare.html and http://www.astro.caltech.edu/ ftod/sleuth.html. 
TABLE 1

TrES-1 Parent Star

\begin{tabular}{|c|c|}
\hline Parameter & Value \\
\hline R.A. & $19^{\mathrm{h}} 04^{\mathrm{m}} 09.8$ (J2000.0) \\
\hline Decl. .......... & $+36^{\circ} 37^{\prime} 57^{\prime \prime}(\mathrm{J} 2000.0)$ \\
\hline$R$ & 11.34 \\
\hline$V \ldots \ldots$ & 11.79 \\
\hline$B-V \ldots \ldots \ldots$ & 0.78 \\
\hline$J \ldots \ldots \ldots \ldots$ & 10.294 \\
\hline$J-H$ & 0.407 \\
\hline$J-K$. & 0.475 \\
\hline Spectrum ..... & K0 V \\
\hline$M_{s} \ldots \ldots \ldots \ldots$ & $0.88 \pm 0.07 M_{\odot}$ \\
\hline$R_{s} \ldots$ & $0.85_{-005}^{+0.10} R_{\odot}$ \\
\hline GSC & $02652-01324$ \\
\hline 2MASS $\ldots \ldots$ & $19040985+3637574$ \\
\hline
\end{tabular}

confirmed planet detected using the TrES network; we refer to the parent star by the same name, since the distinction between planet and star will be clear from context. The star's coordinates, observed characteristics, and index numbers from various full-sky catalogs are given in Table 1 . The $V$ and $B-V$ values come from differential photometry relative to 32 stars with $B$ and $V$ data in SIMBAD; Johnson $R$ magnitudes were obtained from observations of Landolt's standards (Landolt 1992), and the $J H K$ values are from the Two Micron All Sky Survey (2MASS) catalog. The field containing this star was observed by two sites (STARE and PSST) during the summer of 2003, with STARE obtaining 49 good nights of observations and PSST 25. The Sleuth telescope was still under development at that time, and so did not observe this field.

The top panel of Figure 1 shows the near-transit portion of the light curve of TrES-1, folded with a period of 3.030065 days. This curve is a superposition of four full transits and two partial ones, all observed with the STARE telescope. Even though the PSST telescope obtained 25 nights of good observations on the field, it observed no transits of TrES-1 in 2003. This circumstance arose because the orbital period is very nearly an integral number of days, so that for long intervals, transits can be observed only from certain longitudes on the Earth. Although data from PSST played no role in detecting the transits, its data proved essential for a correct determination of the orbital period: we rejected several candidate periods because they implied transit events that were not seen from the western US. TrES-1 is thus a graphic demonstration of the utility of a longitude-distributed network of transit-detection telescopes.

The $R$-band transit seen by TrES has a flat-bottomed shape, a depth of $0.023 \mathrm{mag}$, and a total duration of about $3 \mathrm{hr}$. These characteristics are consistent with expectations for a Jupiter-sized planet crossing a cool dwarf star, but both experience (Latham 2003; Charbonneau et al. 2004) and theory (Brown 2003) show that they are more likely to result from an eclipsing stellar system. Multiple-star systems, in which the eclipsing binary component contributes only a small fraction of the total light, are particularly insidious. Thus, TrES- 1 was one of 16 stars that displayed transitlike events among the 12,000 stars we monitored in its surrounding field. We therefore began an extensive program of observations with larger telescopes, to determine whether the eclipses actually result from a body of planetary mass.

From Table 1, the $J-K$ color of 0.48 suggests a star with spectral type of late G or early K. Digitized Sky Survey images show no bright neighbors within the $20^{\prime \prime}$ radius of a STARE stellar image, and adaptive optics $H$ - and $K$-band imaging with the William Herschel Telescope showed no companion within 2 mag in brightness, farther than 0.3 from the primary star. With its observed $V$ magnitude of 11.79 , and ignoring interstellar ex-

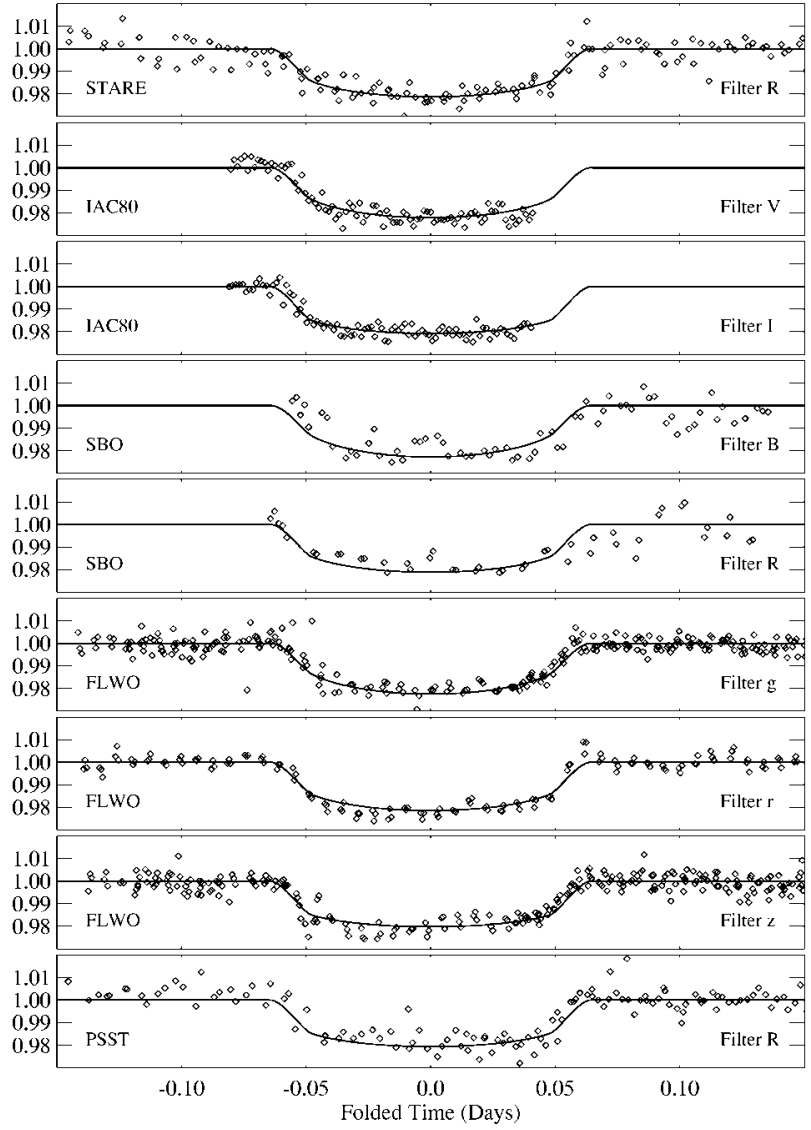

FIG. 1.-Time series photometry used in estimating the radius and inclination of TrES-1, plotted against heliocentric time modulo the orbital period from Table 2. The telescope and filter bandpass used are indicated on each plot. Each set of observations is overplotted with the predicted light curve for that color, given the parameters in Table 2.

tinction, the implied distance to TrES- 1 is about $150 \mathrm{pc}$. Combining this distance with the USNO-B1.0 proper motion of 47 mas $\mathrm{yr}^{-1}$ (Monet et al. 2003) gives a transverse velocity of 26 $\mathrm{km} \mathrm{s}^{-1}$, which is fairly typical for low-mass field stars in the solar neighborhood. Thus, the photometric and astrometric evidence tends to confirm that most of the detected light comes from a nearby dwarf star.

We observed the star using the CfA Digital Speedometers (Latham 1992) at seven different epochs, giving coverage of the full orbital phase. These instruments record $4.5 \mathrm{~nm}$ of spectrum centered on the $\mathrm{Mg} b$ lines, with spectral resolution of about 8.5 $\mathrm{km} \mathrm{s}^{-1}$. For the seven exposures spanning 60 days, we determined a mean velocity of $-20.52 \mathrm{~km} \mathrm{~s}^{-1}$. The average internal error estimate and actual velocity rms achieved were both 0.39 $\mathrm{km} \mathrm{s}^{-1}$, suggesting that any companion orbiting with a 3.03 day period must have a mass smaller than $5 M_{\text {Jup }}$. This conclusion is not firm, however, if there is blending light from a third component. From comparisons of our observed spectra with synthetic spectra calculated by J. Morse using Kurucz models (J. Morse \& R. L. Kurucz 2004, private communication), we estimate that TrES-1 has $T_{\text {eff }}=5250 \pm 200 \mathrm{~K}, \log g=4.5 \pm 0.5$, $v \sin i \leq 5 \mathrm{~km} \mathrm{~s}^{-1}$, and metallicity similar to that of the Sun. The slow rotation is particularly significant, for several reasons: it indicates that the star has not been spun up by tidal interactions with a massive secondary, it forecloses some blending scenarios, and it means that more precise radial velocity measurements can be obtained fairly readily. 


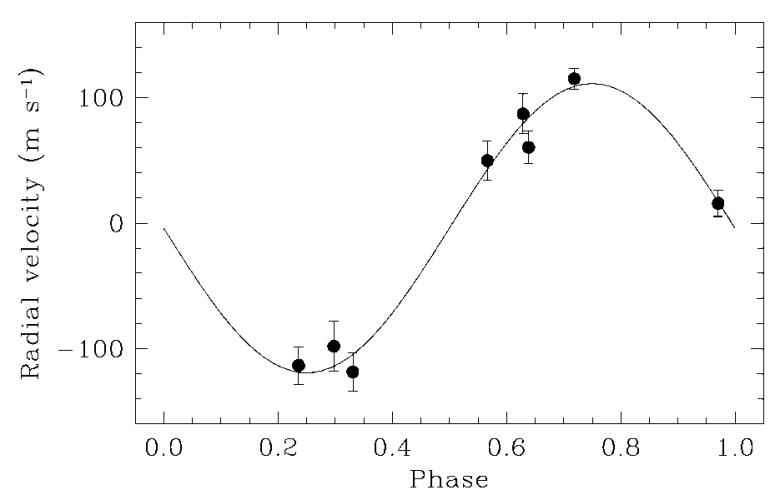

FIG. 2.-Radial velocity observations of TrES-1, overplotted with the bestfit orbit.

We also obtained a moderate-resolution echelle spectrum covering the entire visible wavelength range, using the Palomar $1.5 \mathrm{~m}$ telescope. Based on the comparison of this spectrum with the spectral standards of Montes et al. (1999), we classify the star as $\mathrm{K} 0 \mathrm{~V}$; it shows no sign of a composite spectrum nor of other peculiarities.

Many multiple-star configurations involve components with different colors, which cause the blended eclipses to have colordependent depths. Moreover, the detailed shape of eclipse light curves provides two independent estimates of the secondary's size, relative to that of the primary star. One of these estimates comes from the eclipse depth, and the other from the duration of the eclipse's ingress and egress portions (Brown et al. 2001; Seager \& Mallén-Ornelas 2003). Consistency between these estimates is an indication that blending with light from a third star is not important. We therefore obtained multicolor photometric observations of several transits, using larger telescopes and a variety of filters. At the Instituto de Astrofísica de Canarias (IAC) $80 \mathrm{~cm}$ telescope, we observed a partial transit (missing the egress) with Johnson $V$ and $I$ filters; at the University of Colorado SommersBausch Observatory (SBO) $61 \mathrm{~cm}$ telescope, we observed a full transit with Johnson $B$ and $R$ filters; at the CfA's Fred L. Whipple Observatory (FLWO) $1.2 \mathrm{~m}$ telescope, we observed one full and one partial transit with Sloan $g, r$, and $z$ filters. The PSST telescope also observed four transits in $R$ during the 2004 season. ${ }^{8}$ Figure 1 displays all of these observations, along with a fit to a model, which we shall discuss below. The light curves show no evidence for color dependence of the transit depth (beyond that expected from color-dependent stellar limb darkening), and both the transit depth and the short ingress/egress times are consistent with transits by an object whose radius is a small fraction (less than about 0.15 ) of the primary star's radius.

Detailed modeling of the light curves following Torres et al. (2004b) was carried out in an attempt to explain the observations as the result of blending with an eclipsing binary. We found all plausible fits to be inconsistent with constraints from the CfA spectroscopy. We conclude that TrES-1 is not significantly blended with the light of another star.

On the strength of the foregoing analysis, we obtained precise radial velocity measurements using the $I_{2}$ absorption cell and High Resolution Echelle Spectrometer on the Keck I telescope. Eight observations were collected over a period of 18 days in 2004 July, providing good coverage of critical phases. The data reduction involved modeling of the temporal and spatial varia-

\footnotetext{
${ }^{8}$ The photometric and radial velocity data described in the text are available at http://www.hao.ucar.edu/public/research/stare/data/TrES1.asc.
}

TABLE 2

TrES-1 Planet

\begin{tabular}{ll}
\hline \hline Parameter & \multicolumn{1}{c}{ Value } \\
\hline \multicolumn{2}{c}{ Orbital } \\
\hline$P \ldots \ldots \ldots \ldots$ & $3.030065 \pm 8 \times 10^{-6}$ days \\
$T_{c} \ldots \ldots \ldots$ & $2,453,186.8060 \pm 0.0002(\mathrm{HJD})$ \\
$a \ldots \ldots \ldots \ldots$ & $0.0393 \pm 0.0011 \mathrm{AU}$ \\
$i \ldots \ldots \ldots \ldots$ & $88.5_{-2.2}^{+1.5} \mathrm{deg}$ \\
$K \ldots \ldots \ldots$ & $115.2 \pm 6.2 \mathrm{~m} \mathrm{~s}^{-1}$ \\
\hline \multicolumn{2}{c}{ Physical } \\
\hline$M_{p} \ldots \ldots \ldots$ & $(0.75 \pm 0.07) M_{\mathrm{Jup}}$ \\
$R_{p} \ldots \ldots \ldots$ & $1.08_{-0.04}^{+0.18} R_{\mathrm{Jup}}$ \\
$R_{p} / R_{s} \ldots \ldots$ & $0.130_{-0.003}^{+0.009}$ \\
\hline
\end{tabular}

tions of the instrumental profile of the spectrograph (Valenti et al. 1995) and is conceptually similar to that described by Butler et al. (1996). Internal errors were computed from the scatter of the velocities from the echelle orders containing $\mathrm{I}_{2}$ lines and are typically $10-15 \mathrm{~m} \mathrm{~s}^{-1}$. Figure 2 shows the radial velocity measurements, along with a fit to a sinusoidal variation that is constrained to have the period and phase determined from the photometric data. This constrained fit matches the data well and yields a velocity semiamplitude of $K=115.2 \pm 6.2 \mathrm{~m} \mathrm{~s}^{-1}$. The rms residual of the fit is $14 \mathrm{~m} \mathrm{~s}^{-1}$, in good agreement with the average of the internal errors. Examination of the spectral line profiles in our Keck spectra by means of the bisector spans (Torres et al. 2004a) indicated no significant asymmetries and no correlation with orbital phase, once again ruling out a blend.

\section{DISCUSSION}

For purposes of an initia estimate of the planetary mass and radius, we assumed TrES- 1 to have $T_{\text {eff }}=5250 \mathrm{~K}$ and solar metallicity. By comparing with the accurately known mass and radius of $\alpha$ Cen $\mathrm{B}$, which has a similar $T_{\text {eff }}$ but probably higher metallicity (Eggenberger et al. 2004), and correcting for the assumed metallicity difference of $\delta[\mathrm{Fe} / \mathrm{H}]=-0.2$ using evolutionary models by Girardi et al. (2000), we estimate a stellar mass $M_{s}=0.88 M_{\odot}$ and a radius $R_{s}=0.85 R_{\odot}$. We took limb darkening relations from Claret (2000) and from A. Claret (2004, private communication), for models with solar metallicity, $\log g=4.5$, and $T_{\text {eff }}=5250 \mathrm{~K}$. We assign (somewhat arbitrarily) an uncertainty of $\pm 0.07 M_{\odot}$ to $M_{s}$. We also take $0.80 R_{\odot} \leq R_{s} \leq 0.95 R_{\odot}$, since adequate fits to the photometry cannot be obtained for stellar radii outside this range.

Using the approximate orbital period and the constraints and assumptions just described, we estimated the orbital semimajor axis $a$ and planetary mass $M_{p}$ from the observed stellar reflex velocity and Kepler's laws. We then performed a minimum- $\chi^{2}$ fit to all of the photometry (with errors estimated from the internal scatter of the input data, taken when possible from the out-oftransit data only), to obtain estimates for the planetary radius $R_{p}$ and orbital inclination $i$, and refined estimates for the epoch of transit center $T_{c}$ and for the orbital period $P$. Our best estimates of the planet's orbital and physical parameters are given in Table 2, and the solid curves in Figures 1 and 2 show the fitted photometric and radial velocity variations overplotted on the data.

The error estimates given in Table 2 include errors that follow from our uncertainty in the radius and mass of the parent star (which is assumed to be a main-sequence object), as indicated in Table 1. These uncertainties (especially in $R_{s}$ ) dominate errors in the photometry as regards estimates of $R_{p}$ and $i$. If the stellar radius and mass were known accurately, the uncertainties in 
$R_{p}$ and in $i$ would be smaller by about a factor of 10. Contrariwise, if the star is actually a subgiant (photometric constraints notwithstanding), $R_{p}$ could exceed the upper limit in Table 2. The error in $M_{p}$ arises about equally from the radial velocity measurement precision and from our uncertainty in $M_{s}$.

The mass, orbital radius, and radiative equilibrium temperature of TrES-1 are quite similar to those of HD 209458b, yet the former planet's radius is about $20 \%$ smaller. Indeed, as shown in Figure 3, the radius of TrES-1 is more similar to those of the OGLE planets, and it closely matches current models for irradiated planets without internal energy sources (Chabrier et al. 2004; Burrows et al. 2004). This discrepancy between the radii of HD 209458b and TrES-1 reinforces suspicion that HD 209458b has an anomalously large radius.

The confrontation between theory and observation for this object would be facilitated if the stellar radius and (to a lesser degree) mass could be better constrained. We are undertaking a careful study of the Keck spectra of TrES-1, and we will report improved estimates of the stellar parameters derived from them in a later paper. In the long run, however, a better approach is to obtain improved observations. With space-borne photometry, one can achieve low enough noise to fit for both the planetary and the stellar radius (Brown et al. 2001). Although one still requires a guess for $M_{s}$, the derived planetary properties are much less sensitive to this parameter than they are to $R_{s}$. Similarly, an accurate parallax measurement would imply a useful constraint on $R_{s}$. Thus, TrES-1 may be an attractive target for either groundor space-based interferometric astrometry, since it is relatively bright $(K=9.8)$, and it has several neighbors of similar brightness within a radius of a few arcminutes.

We thank the students and staff of many observatories for their assistance in obtaining the data needed for this study. At FLWO, we thank Perry Berlind, Mike Calkins, and Gil Esquerdo; at the Oak Ridge Observatory, Joe Caruso, Robert Davis, and Joe Zajac; at Izaña/Tenerife, Cristina Abajas, Luis Chinarro, Cristina Díaz, Sergio Fernández, Santiago López, and Antonio Pimienta; at SBO, Christine Predaina, Lesley Cook, Adam Ceranski, Keith Gleason, and John Stocke. We also thank A. Claret for computing limb-

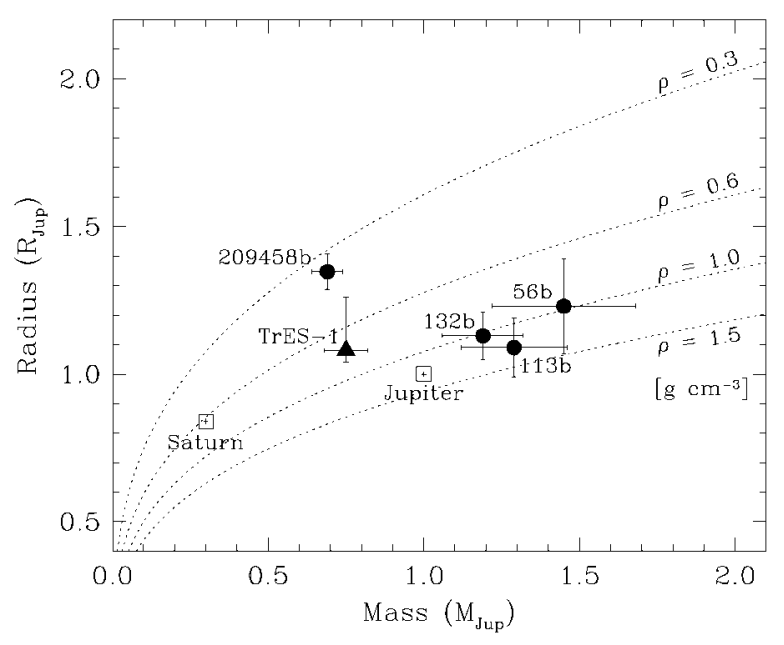

FIG. 3.-Radii of transiting extrasolar planets plotted against their masses. Dashed curves are lines of constant density. Data are from Brown et al. (2001) for HD 209458b, Torres et al. (2004a) for OGLE-TR-56, Bouchy et al. (2004) and Konacki et al. (2004) for OGLE-TR-113, Moutou et al. (2004) for OGLETR-132, and this Letter for TrES-1.

darkening coefficients for the Sloan filter set and J. Morse for helping with models for stellar classification. Partial support for some of this work was provided through NASA's Kepler Project, W. Borucki, principal investigator. The IAC $80 \mathrm{~m}$ telescope is operated by the Instituto de Astrofísica de Canarias in its Observatorio del Teide. This publication makes use of data products from 2MASS, which is a joint project of the University of Massachusetts and the Infrared Processing and Analysis Center/California Institute of Technology, funded by the National Aeronautics and Space Administration and the National Science Foundation. Some of the data presented herein were obtained at the W. M. Keck Observatory, which is operated as a scientific partnership among the California Institute of Technology, the University of California, and the National Aeronautics and Space Administration. The Observatory was made possible by the generous financial support of the W. M. Keck Foundation.

\section{REFERENCES}

Alard, C. 2000, A\&AS, 144, 363

Alonso, R., Deeg, H. J., Brown, T. M., \& Belmonte, J. A. 2004, Astron. Nachr., in press

Auvergne, M., et al. 2003, Proc. SPIE, 4854, 170

Borucki, W. J., et al. 2003, Proc. SPIE, 4854, 129

Bouchy, F., Pont, F., Santos, N. C., Melo, C., Mayor, M., Queloz, D., \& Udry, S. 2004, A\&A, 421, L13

Brown, T. M. 2003, ApJ, 593, L125

Brown, T. M., Charbonneau, D., Gilliland, R. L., Noyes, R. W., \& Burrows, A. 2001, ApJ, 552, 699

Burrows, A., Hubeny, I., Hubbard, W. B., Sudarsky, D., \& Fortney, J. J. 2004, ApJ, 610, L53

Butler, R. P., Marcy, G. W., Williams, E., McCarthy, C., Dosanjh, P., \& Vogt, S. S. 1996, PASP, 108, 500

Chabrier, G., Barman, T., Baraffe, I., Allard, F., \& Hauschildt, P. H. 2004, ApJ, 603, L53

Charbonneau, D., Brown, T. M., Dunham, E. W., Latham, D. W., Looper, D., \& Mandushev, G. 2004, in AIP Conf. Proc. 713, The Search for Other Worlds, ed. S. S. Holt \& D. Deming (Melville: AIP), 151

Charbonneau, D., Brown, T. M., Latham, D. W., \& Mayor, M. 2000, ApJ, 529, L45

Claret, A. 2000, A\&A, 363, 1081

Dunham, E. W., Mandushev, G., Taylor, B., \& Oetiker, B. 2004, PASP, submitted

Eggenberger, P., Charbonnel, C., Talon, S., Meynet, G., Maeder, A., Carrier, F., \& Bourban, G. 2004, A\&A, 417, 235
Girardi, L., Bressan, A., Bertelli, G., \& Chiosi, C. 2000, A\&AS, 141, 371 Henry, G., Marcy, G. W., Butler, R. P., \& Vogt, S. S. 2000, ApJ, 529, L41 Horne, K. 2003, in ASP Conf. Ser. 294, Scientific Frontiers in Research on Extrasolar Planets, ed. D. Deming \& S. Seager (San Francisco: ASP), 361 Konacki, M., Torres, G., Jha, S., \& Sasselov, D. D. 2003, Nature, 421, 507

Konacki, M., et al. 2004, ApJ, 609, L37

Landolt, A. U. 1992, AJ, 104, 340

Latham, D. W. 1992, in IAU Colloq. 135, Complementary Approaches to Double and Multiple Star Research, ed. H. A. McAlister \& W. I. Hartkopf (ASP Conf. Ser. 32; San Francisco: ASP), 110

. 2003, in ASP Conf. Ser. 294, Scientific Frontiers in Research on Extrasolar Planets, ed. D. Deming \& S. Seager (San Francisco: ASP), 409

Monet, D. G., et al. 2003, AJ, 125, 984

Montes, D., Ramsey, L. W., \& Welty, A. D. 1999, ApJS, 123, 283

Moutou, C., Pont, F., Bouchy, F., \& Mayor, M. 2004, A\&A, in press (astro$\mathrm{ph} / 0407635)$

Seager, S., \& Mallén-Ornelas, G. 2003, ApJ, 585, 1038

Struve, O. 1952, Observatory, 72, 199

Torres, G., Konacki, M., Sasselov, D. D., \& Jha, S. 2004a, ApJ, 609, 1071 2004b, ApJ, in press (astro-ph/0406627)

Udalski, A., Pietrzyński, G., Szymański, M., Kubiak, M., Żebruń, K., Soszyński, I., Szewczyk, O., \& Wyrzykowski, Ł. 2003, Acta Astron., 53, 133

Udalski, A., Żebruń, K., Szymański, M., Kubiak, M., Soszyński, I., Szewczyk, O., Wyrzykowski, Ł., \& Pietrzyński, G. 2002a, Acta Astron., 52, 115

Udalski, A., et al. 2002b, Acta Astron., 52, 1

Valenti, J. A., Butler, R. P., \& Marcy, G. W. 1995, PASP, 107, 966 